\title{
Improved outcomes from penile prosthetic implantation surgery may be achieved via a consistent, team-based approach
}

\author{
Jason R. Kovac \\ Men's Health Center, Indianapolis, Indiana 46260, USA \\ Correspondence to: Dr. Jason R. Kovac, MD, PhD, FACS, FRCSC. Men's Health Center, 8240 Naab Road, Suite 220, Indianapolis, Indiana 46260, \\ USA. Email: jkovac@urologyin.com. \\ Comment on: O’Rourke TK Jr, Erbella A, Zhang Y, Wosnitzer MS. Prevention, identification, and management of post-operative penile implant \\ complications of infection, hematoma, and device malfunction. Transl Androl Urol 2017;6:S832-48.
}

Submitted Nov 08, 2017. Accepted for publication Nov 08, 2017.

doi: $10.21037 /$ tau. 2017.11 .03

View this article at: http://dx.doi.org/10.21037/tau.2017.11.03

Advances over the past few decades, in both device technologies and implantation techniques, have significantly decreased complication rates for inflatable penile prosthesis (IPP) surgeries. However, like all operations, nothing is without risk and potential complication. When concerning the treatment of erectile dysfunction, given the elective nature of the surgery, high outcome standards are expected. As such, urologic prosthetic surgeons typically have a lower threshold for cancelling, or delaying cases in situations where optimal requirements are not met.

Many prosthetic surgeons will go to great lengths to minimize the likelihood of post-operative complications. Post-operative infections of $\sim 1-3 \%$, hematoma rates of $\sim 1 \%$ and functional device rates of $\sim 78-85 \%$ at 15 years (1) gives re-assurance that progress continues to be made. Along with this trend, research continues to suggest that prosthetic surgeons with higher case volumes traditionally obtain improved results. Once surgeon technique and experience has been established, other factors must be optimized to ensure best practices.

Primarily, the ability to achieve operative standardization and consistency is the holy grail of prosthetic implantation. Initial measures, assisted by the pre-operative nurses, including setting patient expectations and reliable infusion of intra-venous antibiotics prior to the first incision. The ability of the operating room staff to have all equipment available and "in the room" minimizes delay, while signs posted warning against unnecessary entry into the operating room limits traffic to minimize infection risk.

Establishment of a consistent operating room staff produces both a familiarity with the surgical procedure as well as with others on the team. Resultant faster operating times, improved patient outcomes and higher job satisfaction should all be expected. Utilizing the same "implant team" to do the same surgery, the same way, every time should consistently be expected to produce excellent results. The surgical assist, the scrub technician as well as the IPP device representative all play critical roles in various stages of the procedure. Familiarity allows rapid preparation of the device, accurate performance of each of the procedural steps as well as improved recognition of when something is sub-optimal allowing prompt and rapid intervention.

While hospitals and administrators have been slow to catch on, many are now realizing the importance of the "team" approach to prosthetic surgery. While patient outcomes are likely improved, also equally important is that all team members begin to feel valued for their contributions. By becoming invested in both the procedure and the team, members are more likely to strive for excellence, since they are contributors, rather than simple, passive observers. While difficult to capture accurately, future studies examining a "team-based" approach to prosthetic implantation would be valuable in helping to establish such an approach as the surgical standard of care. 


\section{Acknowledgements}

None.

\section{Footnote}

Conflicts of Interest: The author has no conflicts of interest to declare.

Cite this article as: Kovac JR. Improved outcomes from penile prosthetic implantation surgery may be achieved via a consistent, team-based approach. Transl Androl Urol 2017;6(Suppl 5):S849-S850. doi: 10.21037/tau.2017.11.03

\section{References}

1. O'Rourke TK Jr, Erbella A, Zhang Y, Wosnitzer MS. Prevention, identification, and management of postoperative penile implant complications of infection, hematoma, and device malfunction. Transl Androl Urol 2017;6:S832-48. 